\title{
Correspondence
}

\section{Tuberculosis in children}

Sir,

We were interested to read the two articles recently published in Archives from the Medical Research Council Tuberculosis and Chest Disease Unit ${ }^{1}$ and Packe and Innes. ${ }^{2}$ We were particularly interested in the comments on tuberculous meningitis in children.

We have recently reviewed the cases of tuberculous meningitis seen at the Royal Hospital for Sick Children, Glasgow. ${ }^{3}$ Between 1968 and 1986, 15 children were seen because of this condition. Fourteen were white and only one was Asian. The mean age at presentation was 2 years (12 months to 12 years) and none had received BCG vaccination. In 12 children close contact with other cases of tuberculosis was identified.

It is clear that the practice of offering BCG vaccination to children at 13 years of age was too late to protect the children we studied from acquiring the infection. Routine BCG vaccination of all neonates was stopped in Glasgow in 1974 and since then has been offered only to babies of Asian or Chinese origin. This may partly explain why most cases of tuberculous meningitis seen in our hospital occurred in white children.

We agree with the recommendation of the World Health Organisation that the use of BCG vaccination should be as early in life as possible and stress that any abandonment of such policy must be accompanied by meticulous case reporting and contact tracing if more cases of this serious condition are to be avoided.

\section{References}

${ }^{1}$ Medical Research Council Tuberculosis and Chest Diseases Unit. Tuberculosis in children: a national survey of notifications in England and Wales in 1983. Arch Dis Child 1988;63:266-76.

2 Packe GE, Innes JA. Protective effect of BCG vaccination in infant Asians: a case-control study. Arch Dis Child 1988; 63:277-81.

3 Galea P, Goel KM. Tuberculous meningitis in childhood, a review of 15 cases. Scott Med J 1988;33:205-7.

P Galea and $\mathrm{K}$ M Goel Royal Hospital for Sick Children, Yorkhill,

Glasgow G3 8SJ

\section{Total parenteral nutrition and sepsis}

Sir,

The paper by Dr Beganović and colleagues on total parenteral nutrition and sepsis contributes little to our understanding of either of these issues. ${ }^{1}$ Sepsis was considered proved by positive bacteriology in $13 \%$ of infants receiving total parenteral nutrition and $6 \%$ of those who did not (the percentages given in the table have been miscalculated). Eighty per cent of blood cultures grew Staphylococcus epidermidis, Staphylococcus aureus, Escherichia coli or group B streptococcus, important causes of serious infection in the newborn period, puzzlingly described by the authors as 'fairly benign'.

We are not told the proportion of patients with a central venous catheter for total parenteral nutrition and those fed through a peripheral venous cannula. The effect that this might have had on sepsis rates within the group receiving total parenteral nutrition is of considerable practical importance. Patients receiving total parenteral nutrition were almost certainly more likely than enterally fed infants to have other sources of infection such as arterial catheters, endotracheal tubes, chest drains, etc. The lack of information relating to primary and secondary catheter sepsis makes it impossible to assess objectively the risk of infection related directly to administration of total parenteral nutrition.

The authors conclusion that the advantages of total parenteral nutrition outweigh the disadvantages of sepsis in this group of infants' is extraordinary as no details of the advantages derived from total parenteral nutrition or the morbidity and mortality associated with sepsis are given. The fact that $64 \%$ of the study population received total parenteral nutrition indicates an astonishingly high proportion of neonates in whom enteral nutrition was considered to be contraindicated.

We suggest that the frequent recourse to total parenteral nutrition as highlighted in this study does not reflect 'the fact that the commercially available solutions have been adapted from premature newborn infants' so much as an uncritical approach to the use of a valuable treatment, but one which despite the authors opinions has a more limited application than they imply.

\section{Reference \\ 1 Beganović N, Verloove-Vanhorick SP, Brand R, Ruys JH. Total parenteral nutrition and sepsis. Arch Dis Child 1988;63: 66-7.}

J W L Puntis and I W Booth Institute of Child Health, Edgbaston, Birmingham B16 8ET

Drs Verloove-Vanhorick and Beganović comment:

Dr Puntis misunderstood our paper on total parenteral nutrition. Our aim was, foremost, to provide data on total parenteral nutrition and sepsis in a relatively huge cohort of very low birthweight or very preterm babies. Obviously, in such a nationwide, collaborative epidemiological study in a high risk population a single aspect of perinatal care cannot be recorded exhaustively. Dr Puntis' criticism on lack of certain data seems therefore unwarranted. Some points, however, may easily be clarified. 